\title{
THE DUALISATION METHOD REVISITED: DUALISATION OF PRODUCT LAGUERRE COMPLEXES AS A UNIFYING FRAMEWORK
}

\author{
FRANZ GÄHLER \\ Département de Physique Théorique, Université de Genève, \\ 24 quai Ernest Ansermet, CH-1211 Genève 4, Switzerland \\ and \\ PETER STAMPFLI \\ Institut für Theoretische Physik, Freie Universität Berlin, \\ Arnimalle 14, D-1000 Berlin 99, Germany
}

\begin{abstract}
A general framework based on the dualisation of Laguerre cell complexes is presented, which allows to construct and understand a large variety of quasiperiodic tilings, both new ones and well known old ones. The general framework is illustrated with many examples, which are all based on cell complexes which are products of 2-dimensional complexes. The simple structure of these examples makes it particularly easy to understand how the general procedure works. Yet the examples are sufficiently versatile to exploit the power and flexibility of the method.
\end{abstract}

\section{Introduction}

The dualisation method has been the first method to construct quasiperiodic tilings which makes their quasiperiodicity manifest. Although the Penrose tiling had first been constructed by iterated substitution of the tiles by smaller ones, with subsequent scaling of the tiling, it was only after de Bruijn's construction ${ }^{1}$ by dualisation of pentagrids that the quasiperiodicity of the Penrose tilings was fully understood. This dualisation method had soon been generalized to 3-dimensional tilings with icosahedral symmetry by Kramer and $\mathrm{Neri}^{2}{ }^{2}$ and after the additional impetus due to the discovery of quasicrystals the dualisation method was used to construct quasiperiodic tilings of various other symmetries. 3,4

In its original version, the dualisation method could be applied only to hypercubic lattices, which required in general a lattice of a dimension much higher than necessary to obtain a quasiperiodic tiling with a given point symmetry. For this reason, other methods for the construction of quasiperiodic tilings became more popular, in particular the projection method. The projection method, which is equivalent to the dualisation method, ${ }^{3}$ is somewhat easier to generalize, although on a rather ad hoc basis. Later on however, experience was gained how to use the dualisation method in much more general situations. The key to these developments 
is contained already in the paper of Kramer and Neri. ${ }^{2}$ It consists of their careful distinction between the cubic cell complex $\mathcal{C}$ associated with the hypercubic lattice, and its natural dual complex $\mathcal{C}^{\prime}$, which is also cubic and which therefore mistakenly was identified with $\mathcal{C}$ by many authors. If $\mathcal{C}$ and $\mathcal{C}^{\prime}$ are distinguished, and if it is observed that $\mathcal{C}^{\prime}$ is nothing but the Voronoi cell complex of the hypercubic lattice, it becomes evident how to generalize the dualisation method: $\mathcal{C}^{\prime}$ has to be replaced by the Voronoi complex of the lattice, and $\mathcal{C}$ by its natural dual complex, called Delaunay complex. ${ }^{5}$ This prescription works for any lattice, but for lattices not having an orthogonal basis the two complexes become inequivalent. This construction has been systematically applied ${ }^{6}$ to root lattices, which include lattices with the symmetries of all experimentally observed quasicrystals. It can be further generalized by replacing the lattice by a more general discrete point system, and by replacing the Voronoi complex by a Laguerre complex, ${ }^{7}$ which is a generalized Voronoi complex. These generalizations provide a high degree of flexibility to the method.

In its present formulation, ${ }^{8,7}$ the dualisation method has proved to be a very powerful and flexible tool for the construction of quasiperiodic tilings. It is the purpose of this paper to give a review of some of these recent developments, with special emphasis on a particular class of examples. These examples are quasiperiodic tilings obtained from lattices which are orthogonal direct sums of several identical 2-dimensional lattices. For these lattices the various cell complexes used in the dualisation method are all products of identical 2-dimensional cell complexes, which makes it particularly easy to understand how the method works. Some of these examples have already appeared in different contexts in the literature, others are new. By providing several equivalent formulations of the dualisation method, many examples known from the literature can be cast into a general, unifying framework.

The remainder of the paper is organized as follows. In Sec. 2 a general formulation of the classical method ${ }^{9,8}$ for the dualisation of Voronoi complexes is given. Several different aspects of the method are discussed, in order to make it as widely applicable as possible. The lattices which form the basis of our examples are introduced in Sec. 3. In Sec. 4 Laguerre complexes and their dual Delaunay complexes are defined. Instead of duplicating the mathematical discussion of Laguerre complexes given by Schlottmann, ${ }^{7}$ we provide several examples which serve to illustrate the basic ideas. In Sec. 5, product Laguerre complexes are introduced for the construction of the quasiperiodic tilings. The flexibility of the method is illustrated with a few examples, and some interesting crystallographic aspects of the resulting tilings are discussed. In Sec. 6, a review of the many examples known from the literature is presented, examples which all can be cast into our unifying framework. We conclude with some brief remarks on further generalizations.

\section{The Dualisation Method}

In this section, we give a fairly general definition of the dualisation method, from which it will be easy to make further generalizations. We shall use the mathematical 
concept of cell complexes, which proves particularly useful for our purposes. Several other, equivalent formulations will be given as well.

Suppose that we want to construct a $d$-dimensional quasiperiodic tiling with a $d$-dimensional, non-crystallographic point group $G$. For that purpose, we shall require a $G$-symmetric lattice $\Lambda$ of dimension $n>d$, on which $G$ acts with a faithful representation containing the $d$-dimensional representation with which $G$ is to act on the tiling. The invariant subspace of this $d$-dimensional representation is called external space $V_{E}$, and its orthogonal complement is called internal space $V_{I}$. For the moment we assume that the lattice $\Lambda$ has the minimal dimension compatible with the symmetry $G$, which implies that $V_{I}$ does not contain any lattice vectors. We denote by $\pi$ and $\pi^{\prime}$ the orthogonal projectors on external and internal space, respectively.

The Voronoi complex $\mathcal{V}$ of the lattice $\Lambda$ is defined as follows. For every $x_{1} \in \Lambda$, the Voronoi cell $V\left(x_{i}\right)$ associated with $x_{i}$ is defined by

$$
V\left(x_{i}\right)=\left\{x \in R^{n}|| x-\left.x_{i}\right|^{2} \leq\left|x-x_{j}\right|^{2} \forall x, \in \Lambda\right\} .
$$

It can be shown that the Voronoi cells of a lattice are bounded polytopes. Each Voronoi cell results from the intersection of finitely many half-spaces. The union of all Voronoi cells covers the whole space, and different Voronoi cells may intersect only on their boundaries. The intersection of two or more Voronoi cells, if nonempty, is always an entire $m$-face of all those cells, together with all its boundaries. In this sense the set of all Voronoi cells of a lattice, together with all their boundaries, can be given the structure of a cell complex. We shall call this cell complex the Voronoi complex of $\Lambda$. We note that the Voronoi complex inherits the whole symmetry $G$ from $\Lambda$.

The Voronoi complex $\mathcal{V}$ has a natural dual complex $\mathcal{D}$, called the Delaunay complex. ${ }^{5}$ For each $m$-cell $c \in \mathcal{V}$ its dual $(n-m)$-cell $c^{\star} \in \mathcal{D}$ is defined to be the convex hull of all those $x_{\imath} \in \Lambda$ whose Voronoi cells contain $c$. This notion of duality can be applied to Delaunay complexes as well. We note that $\mathcal{V}$ and $\mathcal{D}$ are both dual to each other. It follows from the definition that if two cells in one complex have a common boundary $b$, then their dual cells in the dual complexes are boundaries of the dual of $b, b^{\star}$. For further details on these cell complexes we refer to Ref. 8 .

Having defined this pair of dual cell complexes we now proceed to the definition of the dualisation method. Let $E$ be a $d$-dimensional plane parallel to external space $V_{E}$. $E$ will be called physical space. We assume that $E$ is in a generic position, i.e., it does not intersect any cells of a dimension smaller than $n-d$ in either complex. The quasiperiodic tiling in $E$ is now defined as follows. Its $d$-dimensional tiles are given by the projections $\pi c$ of those $d$-cells $c \in \mathcal{V}$ whose dual cell $c^{\star} \in \mathcal{D}$ intersects $E$. The same holds for the boundaries of the tiles: they are given by the projections of all those $m$-cells, $m<d$, whose dual $(n-m)$-cells intersect $E$. It is therefore natural to call $\pi^{\prime} c^{\star} \subset V_{I}$ the acceptance domain of the tile $\pi c \subset V_{E}$ : Whenever physical space $E$ intersects the acceptance domain of a tile, the tile is present in the 
tiling. It can be shown ${ }^{8}$ that this prescription yields a tiling of $E$ without any gaps or overlaps. The resulting tiling is $G$-symmetric in the sense that it is transformed by $G$ into a tiling which is locally indistinguishable.

In the case when $V_{I}$ contains some sublattice $\Lambda^{\prime}$ of $\Lambda$, some extra care is needed. We first define a lattice $\Lambda^{\prime \prime}$, which is the projection of $\Lambda$ onto the linear subspace $P$ spanned by $\Lambda^{\prime}$. In order to produce a tiling with the full symmetry, the position of the plane $E$ in $V_{I}$ must have a component in the subspace $P$ which is a position of maximal symmetry of the lattice $\Lambda^{\prime \prime}$. We illustrate this with an example, the generalized Penrose tilings, ${ }^{10}$ obtained from the $5 \mathrm{~d}$ hypercubic lattice $\Lambda$. The sublattice $\Lambda^{\prime}$ generated by the vector $(1,1,1,1,1)$ is entirely contained in $V_{I}$. The projection of $\Lambda$ onto the subspace $P$ containing $\Lambda^{\prime}$ is a lattice $\Lambda^{\prime \prime}$ which is five times denser than $\Lambda^{\prime}: \Lambda^{\prime \prime}$ is generated by the vector $\frac{1}{5}(1,1,1,1,1)$. It is now well known ${ }^{11}$ that generalized Penrose tilings have 10 -fold symmetry if and only if the parameter y takes integer or half-integer values; otherwise the symmetry is only 5-fold. These values of $\gamma$ correspond precisely to positions of $E$ in the space $P$ which are either lattice nodes or centers of unit cells of $\Lambda^{\prime \prime}$, respectively.

The definition of the dualisation method given above is rather that of a projection method: all those cells are projected whose dual cells intersect $E$. This is, however, equivalent to the classical dualisation of a grid. The intersection of the complex $\mathcal{V}$ with $E$ provides a cellular decomposition of $E$, called the grid. Each $m$-cell of the grid corresponds to a $(m+n-d)$-cell of $\mathcal{V}$ which intersects $E$, and thus to a $(d-m)$-cell of $\mathcal{D}$ whose projection is a tile or a boundary of a tile in the tiling. It can be shown that the tiling is actually topologically dual to the grid, and it can be recovered from the grid, since there is a unique $(d-m)$-cell of the tiling corresponding to each $m$-cell of the grid. In certain cases the grid is particularly simple, which makes it easy to recover the tiling from the grid. For cubic lattices, for example, the grid consists of $n$ periodic arrays of $(d-1)$-planes in $E$, and in the examples we shall consider in this paper, the grids will consist of the union of several identical periodic grids which are rotated with respect to each other.

There is a further, equivalent way to describe the construction of a quasiperiodic tiling. As any other quasiperiodic structure, such a tiling can be obtained as a section through an $n$-dimensional periodic structure. This structure, which is itself a tiling of $n$-dimensional space, is obtained by Kramer's klotz construction. ${ }^{12,8}$ We shall therefore call it the klotz tiling. Its tiles are given by $\pi^{-1} c \times \pi^{\prime-1} c^{\star}$, where $c$ runs over all $d$-cells in $\mathcal{D}$, and $c^{\star}$ is the $(n-d)$-cell in $\mathcal{V}$ which is dual to $c$. The same can be done with $m$-cells in $\mathcal{D}$ and their dual cells, although the klotz tile in this case will not have full dimension. If now $E$ intersects a cell $c^{\star} \in \mathcal{V}$, then it also intersects the corresponding klotz tile, and vice versa, and its intersection with that klotz tile is precisely the projection of the tile $c \in \mathcal{D}$. The intersection of $E$ with the set of all klotz tiles therefore is identical to the quasiperiodic tiling defined above. From the fact that the dualisation method described above yields the desired result ${ }^{8}$ it can be concluded that the union of all klotz tiles covers the whole space, and that different klotz tiles intersect only along their faces. 
Finally, we should note that the roles of the two cell complexes $\mathcal{V}$ and $\mathcal{D}$ can as well be interchanged, so that we could have used the projected $(n-d)$-cells in the complex $\mathcal{D}$ as acceptance domains for the tiles, and the projected $m$-cells in $\mathcal{V}$ as the tiles. Since there are, in general, several translationally inequivalent $n$-cells in the complex $\mathcal{D}$, we then have several different, translationally inequivalent acceptance domains for the vertices of the tiling. Apart from this difference, the situation is completely symmetric in $\mathcal{V}$ and $\mathcal{D}$.

\section{Hypercubic and Hyperhexagonal Lattices}

From now on we are only interested in 2-dimensional tilings with $n$-fold symmetry, where we assume $n$ to be even. As is well known (see e.g. Refs. 13-15, Appendix A of Ref. 16), the minimal dimension of a lattice that can give rise to such a tiling is $m=\phi(n)$, where $\phi$ is Euler's totient function. $\phi(n)$ is equal to the number of integers $q$ 's which are coprime with $n$, where $1 \leq q<n$. The generator of $n$ fold symmetry acts with a representation on $\Lambda$ which splits into $m / 2$ 2-dimensional real irreducible representations. These irreducible representations act as rotations by angles of $\frac{2 \pi q}{n}$ on their respective representation spaces, where $q$ runs over the integers which are coprime with $n$ and satisfy $1 \leq q<n / 2$. The first one of these irreducible representations is assumed to act on physical space.

We are now interested in those cases where $\Lambda$ is both of minimal dimension and an orthogonal direct sum of 2-dimensional square lattices or hexagonal lattices. From the latter condition it follows that the cell complexes $\mathcal{V}$ and $\mathcal{D}$ are products of 2-dimensional cell complexes, so that the grids become very simple. One such set of lattices are the hypercubic lattices of dimension $m$, where $m$ is a power of 2. It is well known $n^{11,15}$ that hypercubic lattices of dimension $m$ can give rise to $n$-fold symmetric tilings, where $n=2 m$. Since $n$ is a power of 2 , all odd numbers $q$ are coprime with $n$, and therefore the minimal dimension for a lattice compatible with $n$-fold symmetry is indeed $m=n / 2$. Clearly, such hypercubic lattices are orthogonal direct sums of $m / 2$ square lattices, all with the same lattice constant The generator of $n$-fold symmetry permutes these square lattices cyclically, where it is understood that the last one of the square lattices is mapped back onto the first one with a rotation by an angle of $\pi / 2$.

A second set of such lattices are the so-called hyperhexagonal lattices. ${ }^{15}$ The orthogonal direct sum of $m^{\prime}$ 2-dimensional hexagonal lattices, all with the same lattice constant, is a lattice with $n$-fold symmetry, where $n=6 \mathrm{~m}^{\prime}$. Similarly to the hypercubic case, the generator of $n$-fold symmetry cyclically permutes the hexagonal lattices, mapping the last one back onto the first one with a rotation by an angle of $\pi / 3$. If we choose $m^{\prime}$ such that its prime decomposition contains only factors 2 and 3 , then the hyperhexagonal lattice of dimension $m=2 m^{\prime}$ has the minimal dimension compatible with $n$-fold symmetry, $n=3 m$. In fact, the numbers $q$ which are coprime with $n$ are the odd numbers not divisible by three, which amounts to a third of all numbers, so that we indeed have $\phi(n)=n / 3$. Examples of such lattices with the lowest dimensions are 12-, 18-, 24- and 36-fold symmetric lattices 


\section{Laguerre Cell Complexes and their Duals}

Laguerre cell complexes are a natural generalization of Voronoi cell complexes. The theory of Laguerre complexes and their use in the dualisation method is presented in the contribution by Schlottmann, ${ }^{7}$ so that we do not need to repeat it here. Instead, we only give the definition and subsequently illustrate it with several 2-dimensional examples. This will be sufficient for the present purpose, since we are only interested in Laguerre cell complexes which are products of 2-dimensional complexes.

The Voronoi cell $V\left(x_{i}\right)$ of a lattice point $x_{i}$ consists of all points which are not closer to any other lattice point than they are to $x_{i}$. The idea behind Laguerre complexes is to assign different weights to the lattice points. These weights will control the size of Laguerre cells associated with these lattice points. Suppose therefore that every lattice point $x_{i}$ carries a real weight $w_{i}$. Then, the Laguerre cell of $x_{i}$ is defined by

$$
L\left(x_{i}\right)=\left\{x \in R^{n}|| x-\left.x_{i}\right|^{2}-w_{i} \leq\left|x-x_{j}\right|^{2}-w_{j} \quad \forall x_{j} \in \Lambda\right\} .
$$

In other words, $L\left(x_{i}\right)$ is given by the intersection of the half-spaces $E_{j}$ bounded by the planes which are orthogonal to the vector $x_{j}-x_{i}$ and pass through the point $x_{i}+\ell_{s j}\left(x,-x_{i}\right)$, with

$$
\ell_{i j}=\frac{1}{2}\left(1-\frac{w_{j}-w_{i}}{\left|x_{j}-x_{i}\right|^{2}}\right) .
$$

We note that if all weights $w_{j}$ are identical we obtain the Voronoi complex as a special case (compare Eqs. (1) and (2)). In the Voronoi case the bounding planes all pass through the midpoint between $x_{i}$ and $x_{j}$, which implies that Voronoi cells are always non-degenerate, i.e., they have a positive volume. In contrast to this, a Laguerre cell $L\left(x_{i}\right)$ may be completely empty if the weight $w_{i}$ of the point $x_{i}$ is too small in comparison to the weights of the neighboring points.

For any Laguerre complex a natural dual Delaunay complex is defined ${ }^{7}$ : As in the Voronoi case, the cell which is dual to a cell $c$ in the Laguerre complex consists of the convex hull of all those $x_{j}$ whose Laguerre cells contain $c$.

Since we are interested in periodic Laguerre complexes only we must assign the weights to the lattice points in a periodic way, and in order to obtain the desired symmetry of the quasiperiodic tiling we must also maintain the symmetry of the lattice. The simplest possibility for this assignment is to choose a sublattice $\Lambda^{\prime}$ of $\Lambda$ with the same symmetry as $\Lambda$, and to assign the same weight to all points which belong to the same coset in $\Lambda / \Lambda^{\prime}$. Moreover, those cosets which form an orbit under the point group of $\Lambda^{\prime}$ also must have the same weights.

The simplest example is a square lattice $\Lambda$, where we choose for $\Lambda^{\prime}$ the sublattice of even sites. The coset space $\Lambda / \Lambda^{\prime}$ consists of two elements, containing the even and odd vertices, respectively. These two cosets both have full symmetry with respect to the point group of the even sublattice, so that each of them forms a full orbit under the point group of $\Lambda^{\prime}$. Therefore, we have in total two different 


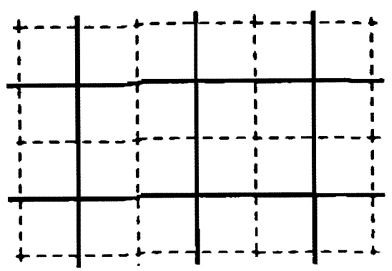

(a)

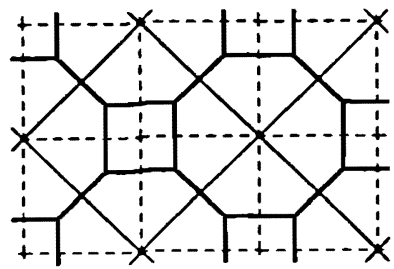

(c)

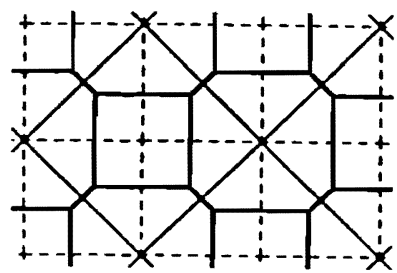

(b)

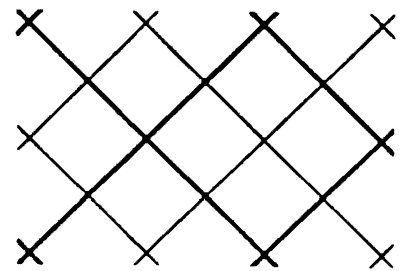

(d)

Fig. 1. Different Laguerre complexes (thick lines) and their dual Delaunay complexes (thin lines), based on a square lattice. The lattice points are divided into two classes with different weights.

weights, $w_{0}$ for the even sites, and $w_{1}$ for the odd sites. For convenience, we fix $w_{0}$ and vary $w_{1}$ relative to $w_{0}$. The various stages of this process are shown in Fig. 1. If $w_{0}=w_{1}$, we obtain the standard Voronoi complex (Fig. 1a, thick lines) and its Delaunay complex (Fig. 1a, thin dashed lines). As soon as the weight $w_{1}$ gets smaller than $w_{0}$, new lines in the Delaunay complex appear (Fig. 1b, thin solid lines), and the even Laguerre cells become octagons, whereas the odd ones remain squares. Both complexes therefore change their topology. If we further decrease $w_{1}$, we pass through one of the Archimedian semi-regular tesselations, which occurs when all edge lengths of the Laguerre cells are identical (Fig. 1c), and finally the odd Laguerre cells disappear completely (Fig. 1d), as well as some of the lines in the Laguerre complex (the dashed ones). Thus we come back to a square Voronoi complex, the one of the sublattice of even sites. Instead of decreasing $w_{1}$, we could also have increased $w_{1}$; in this case the roles of the even and odd sublattices simply would have been interchanged.

As a second example we take a hexagonal lattice $\Lambda$, with a hexagonal sublattice $\Lambda^{\prime}$ of index 3 in $\Lambda$. The three cosets form two orbits under the point group, one containing the sites of $\Lambda^{\prime}$, the other the two other cosets, which are permuted by the point group. We therefore again have two weights $w_{0}$ and $w_{1}$. The different situations which occur when $w_{1}$ is varied with respect to $w_{0}$ are shown in Fig. 2. If both weights are equal, we obtain the Voronoi complex of $\Lambda$, which is a regular net of hexagons, and its Delaunay complex, a regular triangular net (Fig. 2a). We note that there are two translationally inequivalent classes of triangles in the Delaunay complex. If $w_{1}$ is increased, the topology of the two complexes does not change 


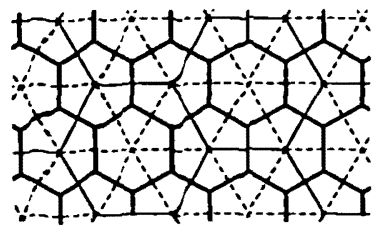

(a)

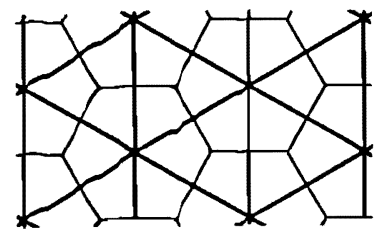

(c)

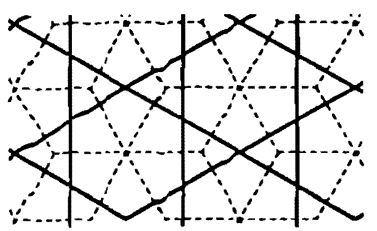

(e)

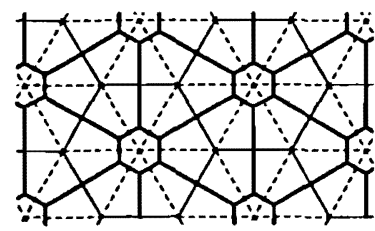

(b)

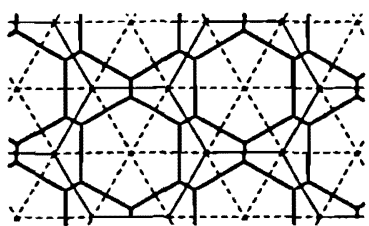

(d)

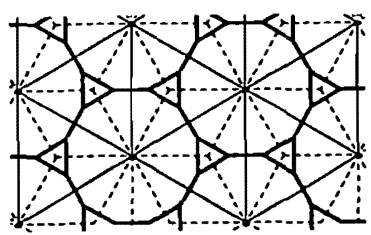

(f)

Fig. 2. Different Laguerre complexes (thick lines) and their dual Delaunay complexes (thin lines), based on a hexagonal lattice. The lattice points are divided into two classes with different weights.

immediately, but the Laguerre cells associated with the sites in the lattice $\Lambda^{\prime}$ shrink (Fig. 2b). When they disappear completely, the Laguerre complex has become a regular triangular complex (Fig. 2c). The centers of its cells are on the two cosets which form a single orbit under the point group of $\Lambda^{\prime}$. At the same time, the corresponding Delaunay complex has become a regular hexagonal complex. These two complexes therefore have exchanged their roles.

If, instead, $w_{1}$ is decreased (Fig. 2d), we pass through a situation where the Laguerre complex is a Kagomé net (Fig. 2e), one of the Archimedian semi-regular tesselations. If we further decrease $w_{1}$, we pass through another Archimedian tesselation (Fig. 2f), where the big Laguerre cells have become dodecagons, before the small Laguerre cells finally completely disappear. We have then arrived at the same situation as shown in Fig. 2c, but this time the hexagon net is the Laguerre complex, and the triangular net is the Delaunay complex.

Instead of breaking, by different weights, the translational symmetry of the lattice to some sublattice, further points could be added to each unit cell in a $G$ symmetric way, and weights could be assigned to them which respect the symmetry. 
All the examples discussed above can be understood in this way. Adding further points is in fact a more flexible method, in the sense that there is more freedom for the positions of these points. As an example, we again take a hexagonal lattice. As one class of points we choose the lattice points, and as two other classes the centers of the triangles and the centers of the bonds, respectively. The centers of the triangles fall into two translationally inequivalent subclasses, to which the same weight must be assigned, since they are permuted by the point group. The bond midpoints fall into three classes and must also all have the same weight. In this example, we have therefore three different weights, so that the space of all possible Laguerre complexes has dimension two (adding the same constant to all weights does not change the Laguerre complex). Some of the different possibilities are shown in Fig. 3. The vertex positions with different weights are shown in black, grey and white, respectively. Thick lines are in the Laguerre complex, thin lines in the Delaunay complex. Between Figs. 3b-3d only the weight of the triangle centers is varied relative to the other two weights. We note that in Fig. 3b an Archimedian tesselation is obtained as the Laguerre complex.

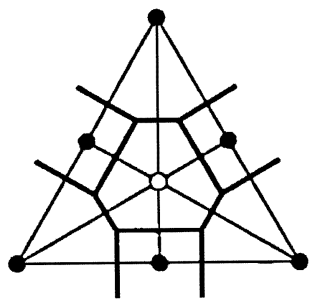

(a)

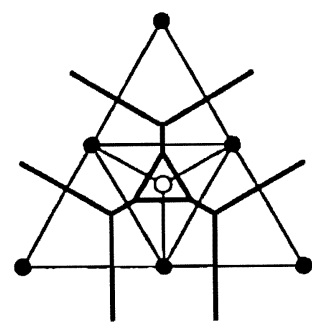

(c)

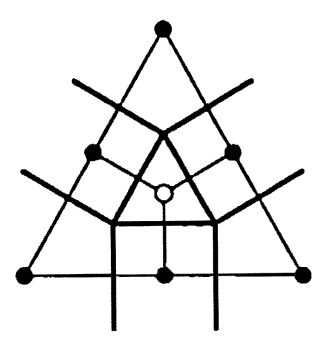

(b)

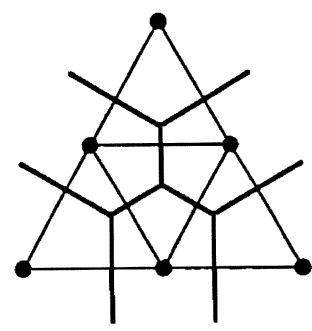

(d)

Fig. 3. Different Laguerre complexes (thick lines) and their dual Delaunay complexes (thin lines). based on a hexagonal lat tice. Further points have been added to the unit cell. Points with different weights are shown in black, grey and white, respectively. 


\section{Examples of Tilings Obtained from Product Laguerre Complexes}

In this section we combine the results of the previous two sections, and then apply the dualisation method to the resulting complexes. We first describe the case of the Voronoi complex, and then generalize to Laguerre complexes. We note that if the lattice $\Lambda$ is an orthogonal direct sum of 2-dimensional lattices, then its Voronoi complex and the associated Delaunay complex are the products of the corresponding Voronoi and Delaunay complexes of the 2-dimensional sublattices. If $\Lambda$ is a sum of $m$ 2-dimensional lattices, then the set of all boundaries of its Voronoi complex is given by the union of $m$ sets, where each set is the product of the Voronoi net of one of the 2-dimensional lattices with all the 2-planes containing the other 2-dimensional lattices. A corresponding statement holds true for the Delaunay complex. Therefore, each of the two complexes is given by the union of $m$ arrays of 'tubes', where the structure of these arrays is given by the corresponding 2dimensional complexes.

Recall now that the generator of $n$-fold symmetry cyclically permutes the 2 dimensional lattices. Furthermore, there is a subgroup of the point group of order 4 or 6 , depending on whether we consider a hypercubic or a hyperhexagonal lattice, which leaves each of the 2-dimensional lattices separately invariant. As the physical space $E$ is parallel to an invariant subspace of the point group, and since the point group acts faithfully on $E$, we must conclude that the intersection of the physical space $E$ with any of the arrays of tubes mentioned above must have the same symmetry as the corresponding 2-dimensional complex. Since all the 2-dimensional complexes which occur are identical, all such intersections must be identical, too, except that they are rotated with respect to each other by angles $\frac{2 \pi}{n}$. In fact, the intersection of $E$ with the Voronoi complex consists of the union of $m$ nets which are similar to the Voronoi nets of the 2-dimensional lattices, rotated with respect to each other by angles $\frac{2 \pi}{n}$. Their relative positions are determined by the position of physical space $E$. Correspondingly, a similar statement holds true for the Delaunay complex. In summary, in the hypercubic case both the intersection of $E$ with the Voronoi and the Delaunay complex are unions of $m$ square nets, whereas in the hyperhexagonal case the intersection of $E$ with the Voronoi complex is the union of $m$ regular hexagon nets, and the intersection of $E$ with the Delaunay complex is a union of $m$ triangular nets. These product cell complexes therefore yield particularly simple grids, which are given by the union of several identical periodic grids.

The same procedure can now be executed with product Laguerre complexes. Each vertex position in the $n$-dimensional structure is given as the sum of the position vectors of one vertex in each of the different 2-dimensional structures. The weight of a vertex in the $n$-dimensional structure is the sum of the weights of these vertices in the 2-dimensional structures. With this choice the $n$-dimensional Laguerre complex is identical to the product complex of the corresponding 2dimensional Laguerre complexes. As above, if we cut such a Laguerre complex with the physical space $E$, we obtain a grid which is the union of $m$ nets which are all similar to the net of all boundaries in the 2-dimensional Laguerre complex. 
Let us now illustrate by an example what changes occur in the tilings obtained by the dualisation method, if the weigths of the Laguerre cells are varied. We take the sum of two hexagonal lattices, which has dodecagonal symmetry. In order to prevent that the topologies of the complexes change immediately, we start with the situation of Fig. 2a. Let us first choose the Laguerre complex as the complex from which the tiles are projected, and the Delaunay complex as the complex which provides the acceptance domains of the tiles and produces the grid. The tiling which is obtained when both weights are the same is shown in Fig. 4a. If the weight $w_{1}$ is then increased, we pass to the situation of Fig. $2 \mathrm{~b}$. During this process, the grid does not change, since the Delaunay complex remains stable. The tiling which is dual to the grid therefore does not change its topology. Instead, its tiles, which are projected 2-cells from the Laguerre complex, which does change, are continuously deformed. Such a tiling is shown in Fig. $4 \mathrm{~b}$, where the ratio of two edge lengths of the tiles was chosen equal to $1 / 2$. It is easily verified that the tilings of Figs. $4 a$ and $4 \mathrm{~b}$ have indeed the same topology.

If the roles of the two complexes are exchanged, the tiles obtained from the stable Delaunay complex remain the same. The grid, however, which is produced by the varying Laguerre complex, changes its topology, and so does the tiling dual to the grid. In Fig. 5 it is demonstrated how some of the tiles are reshuffled when we pass from equal weights (Fig. 5a) to different weights (Fig. 5b). In Fig. 5b the ratio of the lengths of the two line segments in the periodic grids was chosen equal to $4 / 5$, so that those grids are close to regular hexagon grids, and only few tiles are reshuffled. The effect can most easily be seen at places where several $30^{\circ}$ rhombi meet.

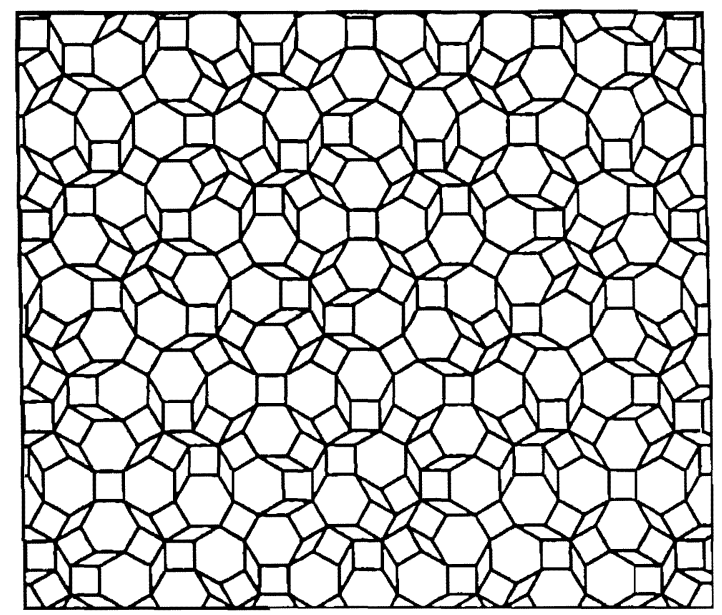

Fig. 4a. Quasiperiodic tiling dual to the union of two triangular nets, with tiles projected from the Voronoi complex. 


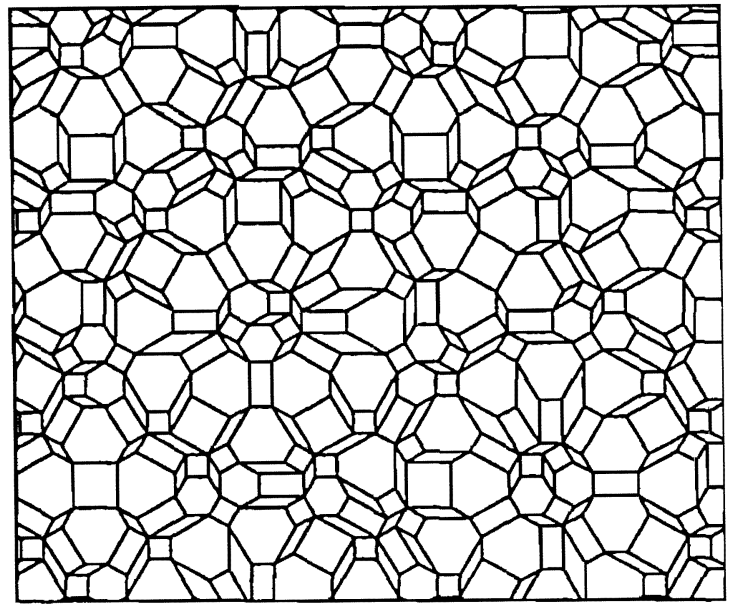

Fig. 4b. Quasiperiodic tiling dual to the union of two triangular nets, with tiles projected from a Laguerre complex close to the Voronoi complex. The topology of the tiling is the same as in Fig. 4a.

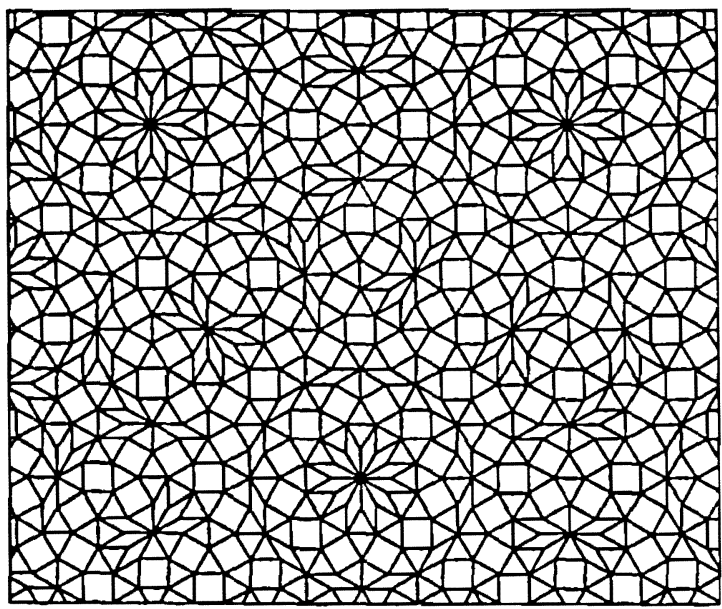

Fig. 5a. Quasiperiodic tiling dual to the union of two hexagon nets, with tiles projected from the Delaunay complex.

In Fig. 6, Laguerre complexes of the type of Fig. $2 \mathrm{~b}$ have again been used for the grid, but this time the small hexagons are very small. The ratio of the two edge lengths of the asymmetric hexagons is only $1 / 8$, so that the grid is close to a grid composed of triangular nets. Figure 6 therefore should be compared with Fig. 4 a. These two tilings are indeed very similar. The opening of the small hexagons adds new lines and vertices (note that new meshes have opened in the grid), which divide 


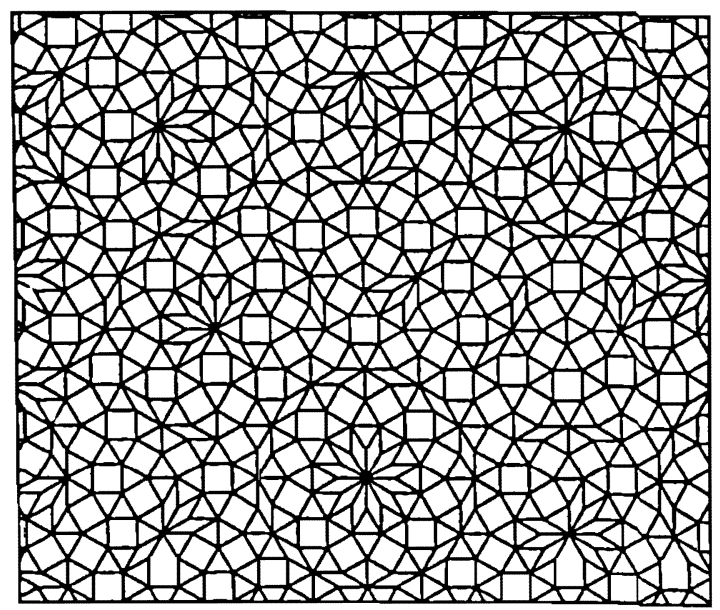

Fig. 5b. Quasiperiodic tiling dual to the union of two slightly distorted hexagon nets (see Fig. 2b), with tiles projected from the Delaunay complex. Only few tiles have been reshuffled with respect to Fig. 5 a.

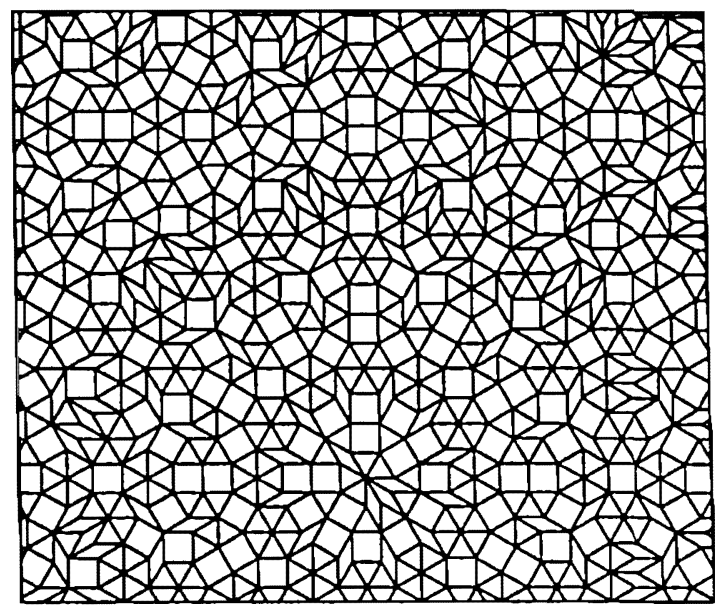

Fig. 6. Quasiperiodic tiling dual to the union of two strongly distorted hexagon nets (see Fig. 2b), with tiles projected from the Delaunay complex. This tiling is similar to the tiling shown in Fig. $4 a$.

the hexagonal tiles from Fig. $4 \mathrm{a}$ into six triangles, but apart from these hexagons only relatively few tiles have changed. This can well be seen in the almost symmetric region centered at the lower middle of the picture.

An interesting feature of these $n$-dimensional Lagusrre complexes is that they have a very rich crystallography. There are in general several classes of translationally inequivalent cells in the complex. Since the whole complex is $G$-symmetric, the 
set of such classes decays into entire orbits under the point group. Cells associated with a vertex $x$ must have the site symmetry of that vertex.

For our examples, which are product complexes of several complexes with 4 - or 6-fold symmetry, it is in fact very easy to determine the symmetry of the different cells, and how these cells form orbits under the point group. Let us illustrate this with an example, the product of two Laguerre complexes of the type shown in Fig. 1b. In the 2-dimensional complex there are two classes of cells, squares and octagons, which both are at positions with 4-fold symmetry, and which therefore are also 4-fold symmetric. Both classes form an entire orbit of their own. The cells of the product Laguerre complex are products of such cells. Clearly, products of two squares and products of two octagons will have 8-fold symmetry, and are situated at the two 8-fold symmetric positions of the 4-dimensional hypercubic lattice, the lattice nodes and the body centers, respectively. Mixed products of one square and one octagon will have only 4 -fold symmetry. Using the results of $\mathrm{Niizeki}^{17}$ on the points of special symmetry for octagonal lattices, it is easily verified that these cells are indeed at positions of 4-fold symmetry, and that these two classes of positions form an entire orbit under the point group. A similar analysis can be carried out also with the more complicated cases, with more than two inequivalent cells in the 2-dimensional complex.

The relevant site symmetry of a vertex is in fact the symmetry of its acceptance domain, i.e., the symmetry of the projection of its dual cell onto internal space. The symmetry of this acceptance domain can easily be deduced for product cells. For our examples, the acceptance domain is simply the vector sum of the 2-dimensional cells from which the $n$-dimensional cell is built. The acceptance domains are often used as 'atomic surfaces', by placing a hypersurface parallel to internal space and of the size and shape of the acceptance domain at the corresponding vertices. If a Laguerre complex is used for the acceptance domains, and if the weights are varied, then the size and shape of these atomic surfaces will vary, but the positions of these 'atoms' will not change in general. In contrast to this, if the Delaunay complex is used for the acceptance domains, then the size and shape of the atomic surfaces does not change, but they wander around when the weights are varied.

\section{Discussion and Conclusion}

In the previous sections we have developed the dualisation method for product Laguerre complexes and their dual Delaunay complexes. This also comprises the special case of Voronoi complexes. In this way, a large variety of different cases can be treated within the same framework. Many of the special cases covered by this framework have previously appeared in the literature in various contexts. It is therefore time to present a survey of all these special cases.

The case of the Voronoi complex of a hypercubic lattice is in fact a classical one. $^{3,4}$ Dualisation of hypercubic lattices had been used well before anyone used the concept of Voronoi cells in this context. It is, however, only for the special 
case in which the dimension of the lattice is a power of 2 that the hypercubic lattice actually has minimal dimension. If the dimension is not minimal, a few subtleties arise. In order to obtain a tiling of the desired symmetry, the position of physical space $E$ in $V_{I}$ is restricted to a subset of $V_{I}$ with lower dimension. This had been largely unknown until the beautiful analysis of this problem by Niizeki." For instance, he showed that if a 12-fold symmetric tiling was to be obtained from a 6-dimensional hypercubic lattice, then the physical space must be chosen such that the grid is either the union of two triangular grids or the union of two Kagomé grids. Both cases are in fact covered by our formalism, using a lattice of minimal dimension. There is no advantage in going to lattices of a dimension higher than required by the symmetry. Niizeki ${ }^{11}$ applied the same argument also to tilings of 18-fold symmetry obtained from hypercubic lattices of dimension 9. Also in this case, only grids which are unions of either three triangular nets or three Kagomé nets can be used to produce tilings of full symmetry.

The tiling of Fig. 4a, which is the dual of a double triangular grid, had first been considered by Socolar ${ }^{\mathrm{a}}$ (see also Ref. 18). It was first published by Niizeki, ${ }^{19}$ however, who discovered it independenly. Dualisation of double hexagonal nets (Fig. 5a) had been applied for the first time in Ref. 20, on a somewhat empirical basis. The theory on which this method is based subsequently was given in Ref. 9, where also the dualisation of general Voronoi complexes was introduced. The general case of tilings which are dual to multiple periodic grids was treated by Niizeki. ${ }^{21}$ In particular, he considered multiple hexagonal, triangular and Kagomé nets to produce tilings with 12- and 18-fold symmetry.

Dualisation of multiple Archimedian semi-regular tesselations, such as those shown in Figs. 1a, 1c, 2a, 2c, 2e, 2f and 3b, had first been considered in Ref. 22. All Archimedian tesselations can in fact be viewed as Laguerre complexes of certain periodic point systems with appropriate weights, and are therefore fully covered by the general theory presented here. In the same paper, ${ }^{22}$ interpolations between different Archimedian tesselations were introduced. In this way, the tiling of Fig. $4 \mathrm{~b}$ has been constructed for the first time. Such interpolations appear naturally in Laguerre complexes, when the weights of the points are varied.

Finally, we present an outlook on further possibilities. In the case of a Voronoi complex, or more generally a Laguerre complex, and its dual Delaunay complex, the general theory of Schlottmann ${ }^{7,8}$ guarantees that the dualisation method always can be applied, and produces a tiling without gaps or overlaps. A prorn, the dualisation method is by no means restricted to such cases, however. We might as well consider a general pair of cell complexes dual to each other, and use the same recipe for dualisation. The only problem which might arise then are possible overlaps of tiles. Whether there are such overlaps can be determined by local inspection of the gnd Each mesh in the grid corresponds to a vertex, and the vertices at the boundary of the mesh determine the tiles that touch the vertex. By inspection of all possible 
types of meshes in the grid it can therefore be determined whether or not overlapping tiles occur. For instance, one may build product complexes from the pair of dual complexes given in Fig. 7 , in which dual tiles are even not orthogonal, and apply the dualisation method to it. Within certain limits, this indeed yields quasiperiodic tilings without overlaps. If the smaller angle of the rhombuses in Fig. 7 is larger than $\pi / 4$, both the product of square complexes and the product of square-rhombus complexes can be used for either role, i.e., they can both provide the tiles or the grid. On the other hand, if the smaller angle of the rhombuses is smaller than $\pi / 4$, then the hypercubic complex must be used for the grid, for otherwise overlapping tiles will occur. Precisely this example, with the cubic complex used for the grid, has been used in Ref. 23 to produce a tiling with non-symmorphic space group symmetry.

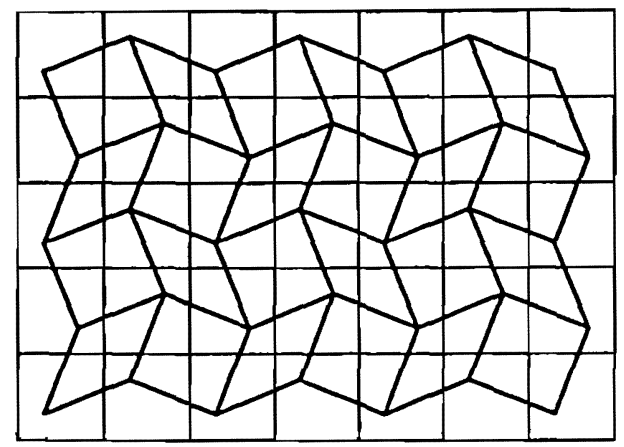

Fig. 7. A pair of dual cell complexes, for which dual cells are not orthogonal.

\section{Acknowledgments}

This work originated from inspiring discussions with Hans-Ude Nissen. We would like to thank him for his continuous encouragement, without which this work would not have been done.

\section{References}

1. N. G. de Bruijn, Proc. Kon. Ned. Akad. Wet. Ser. A, 43, 39, 53 (1981).

2. P. Kramer and R. Neri, Acta Cryst. A40, 580 (1984).

3. F. Gähler and J. Rhyner, J. Phys. A19, 267 (1986).

4. N. G. de Bruijn, J. Physique 47, C3-9 (1986).

5. J. H. Conway and N. J. A. Sloane, Sphere Packings, Lattices and Groups (Springer, New York, 1988).

6. M. Baake, D. Joseph, P. Kramer, and M. Schlottmann, J. Phys. A23, L1037 (1990).

7. M. Schlottmann, this volume, p. 1351.

8. P. Kramer and M. Schlottmann, J. Phys. A22, L1097 (1989).

9. V. E. Korepin, F. Gähler and J. Rhyner, Acta Cryst. A44, 667 (1988). 
10. A. Pavlovitch and M. Kléman, J. Phys. A20, 687 (1987).

11. K. Niizeki, J. Phys. A21, 3333 (1988).

12. P. Kramer, Mod. Phys. Lett. B1, 7 (1987); P. Kramer, Int. J. Mod. Phys. B1, 145 (1987); P. Kramer, J. Math. Phys. 29, 516 (1988).

13. C. Herrmann, Acta Cryst. 2, 139 (1949).

14. H. Hiller, Acta Cryst. A41, 541 (1985).

15. K. Niizeki, J. Phys. A22, 193 (1989).

16. M. Baake, D. Joseph and M. Schlottmann, Int. J. Mod. Phys. B5, 1927 (1990).

17. K. Niizeki, J. Phys. A22, 4281 (1989).

18. J. E. S. Socolar, Phys. Rev. B39, 10519 (1989).

19. K. Niizeki, J. Phys. A21, 2167 (1988).

20. P. Stampfli, Helv. Phys. Acta 59, 1260 (1986).

21. K. Niizeki, J. Phys. A22, 1871 (1989).

22. P. Stampfli, in Quasicrystals, Networks, and Molecules of Fivefold Symmetry, ed. I. Hargittaj (VCH, New York, 1990).

23. D. R. Rabson, T. L. Ho and N. D. Mermin, Acta Cryst. A44, 678 (1988). 